\section{A Ripple Free Sampled-Data Robust Servomechanism Controller Using Exponential Hold}

Yung-Chun Wu and Nie-Zen Yen

\begin{abstract}
In a general command tracking and disturbance rejection problem, it is known that a sampled-data controller using zero-order hold may only guarantee the asymptotic tracking at the sampling instances, but cannot smooth out the ripples between the sampling instances. In this paper, a sampled-data robust servomechanism controller using exponential hold is developed for guaranteeing the asymptotic tracking not only at, but also between, the sampling instances. In this development, a so called "internally-reducible" condition to characterize a class of robust servomechanism controllers is derived first, then the proposed controller is shown to be contained in this class. Generally speaking, a sampled-data structure using exponential hold can provide more design freedoms so that it tends to simplify the construction of a robust servomechanism controller and facilitate the implementation on digital computers. An example for a dc motor control is presented to illustrate the advantages of this approach.
\end{abstract}

\section{INTRODUCTION}

The robust servomechanism problem of a multivariable linear timeinvariant system has been widely considered in the literature. In this problem it is desired to obtain a so-called "robust servomechanism controller" [3], [7] to guarantee the asymptotic tracking and disturbance rejection in the permission of plant variations. In many cases, one may prefer to implement a robust servomechanism controller in digital rather than analog form, especially when only discrete output measurements are dealt with or when the control scheme is to be implemented on a digital computer.

It is known that if the steady state of either the reference input or the disturbance is not constant (e.g., ramps, sinusoids, polynomials, etc.), then a sampled-data controller using zero-order hold will give rise to ripple error. That is, the intersampling error exists and never decays, although the steady-state error is zero at the sampling instances. More seriously, if the sampling rate is slow, then the ripple error may even become unacceptable. The ripple error resulted from this structure has been illustrated by Franklin and Emami [1]; they have shown that the continuous internal model principle is a necessary and sufficient condition for guaranteeing the ripple-free tracking.

To avoid the occurrence of the undesired ripple error, a hybrid method which uses a preparatory analog internal model followed by a digital stabilizing compensator is usually employed [1], [2]. Although such a hybrid controller will guarantee the asymptotic ripple free tracking, a high order single-rate digital compensator may be required since the digital compensator has to stabilize the augmented system comprising the plant and the preparatory analog internal model.

In this paper, a sampled-data robust servomechanism controller combining a discrete-time internal model and an exponential hold is developed. The combination acts as a continuous-time internal model, so that the desired ripple free tracking can be achieved. The developed controller structure is described in a rather general form. A distinctive feature of the structure is that it can lead to a closed-loop system expressible in the dual form of complete state feedback. This allows us to choose the parameters more conveniently (e.g., pole placement or optimal design approach).

Manuscript received June 20, 1990; revised July 1, 1991 and July 19, 1992. N.-Z. Yen is with the Institute of Electronics, National Chiao Tung University, 1001 Ta Hsueh Road, Hsinchu, Taiwan, 30050, R.O.C.

Y.-C. Wu is with the Department of Control Engineering, National Chiao Tung University, 1001 Ta Hsueh Road, Hsinchu, Taiwan, 30050, R.O.C. IEEE Log Number 9400375.
Throughout this paper, the following notations will be used.

$I_{n}=$ the $n \times n$ identity matrix.

$O_{n \times m}=$ the $n \times m$ zero matrix (or simply $O_{n}$ if $n=m$ ).

$\Theta_{m}=$ the zero element of $R^{m}[t]$, the space of all $m \times 1$ time functions.

eig $(\#)=$ the set of eigenvalues of a square matrix \#.

$\exp (\#)=$ the exponential of a square matrix \#.

$[\#]^{\top}=$ the transpose of a matrix \#.

$\mathbb{C}^{+}=$the unstable right-half complex plane including the imaginary axis.

$R^{n \times m}$ (or $\left.C^{n \times m}\right)=$ the set of all real (or complex) matrices of dimension $n \times m$.

$R^{n \times m}[0 T)=$ the set of piecewise continuous functions from $[0 T)$ into $R^{n \times m}$

\section{Preliminary}

Consider the linear time-invariant system

$$
\begin{gathered}
\dot{x}(t)=A x(t)+B u(t)+F d(t) \\
y(t)=C x(t)+G d(t) \\
e(t)=y(t)-r(t)
\end{gathered}
$$

where $x \in R^{n}$ is the state of the system, $u \in R^{m}$ is the input of the system, $y \in R^{m}$ is the output of the system which is measurable and to be regulated, $d \in R^{v}$ is the disturbance, $r \in R^{m}$ is the reference input, and $e \in R^{m}$ is the tracking error. Assume the reference input $r$ and the disturbance $d$ satisfy the following models:

$$
\begin{gathered}
\dot{x}_{r}(t)=A_{r} x_{r}(t) \\
r(t)=C_{r} x_{r}(t)
\end{gathered}
$$

and

$$
\begin{gathered}
\dot{x}_{d}(t)=A_{d} x_{d}(t) \\
d(t)=C_{d} x_{d}(t)
\end{gathered}
$$

where $x_{r} \in R^{m_{r}}, x_{d} \in R^{m_{d}}$, $\operatorname{eig}\left(A_{r}\right) \subset \mathbb{C}^{+}$and $\operatorname{eig}\left(A_{d}\right) \subset \mathbb{C}^{+}$ The system (1) is said to have no transmission zero at the eigenvalues of $A_{d}$ and $A_{r}$ if

$$
\operatorname{rank}\left[\begin{array}{cc}
-s I_{n}+A & B \\
C & O_{m}
\end{array}\right]=n+m, \forall s \in\left\{\operatorname{eig}\left(A_{r}\right) \cup \operatorname{eig}\left(A_{d}\right)\right\} \text {. }
$$

Now, the robust servomechanism problem [4], [7] can be stated alternatively as obtaining a controller to satisfy the following definition (in this paper, only the class of error-driven robust servomechanism controllers is considered).

Definition I: A controller $u=f(e)$ (i.e., with input $e$ and output $u$ ) is a robust servomechanism controller of system (1) if it satisfies the following three conditions:

Condition 1) The resultant closed-loop system is asymptotically stable. That is if $x_{d}(t) \equiv 0$ and $x_{r}(t) \equiv 0$, then $x(t) \rightarrow 0, x_{c}(t) \rightarrow 0$, and $u(t) \rightarrow 0$ as $t \rightarrow \infty$ (where $x_{c}$ denotes the controller state).

Condition 2) The asymptotic tracking action occurs, i.e., $e(t) \rightarrow$ 0 as $t \rightarrow \infty$ for any $x(0), x_{r}(0), x_{d}(0)$ and the initial controller state $x_{c}(0)$.

Condition 3) Condition 2 is true for any plant variations in $A, B, C, F, G, C_{r}$, and $C_{d}$ as long as Condition 1 remains true. 
A general structure of a linear time-invariant robust servomechanism controller has been characterized by Davison et al. [3]-[5]. A well-known criterion of a robust servomechanism controller is the so-called "continuous internal model principle" [8], [6], [1]. Since a robust servomechanism controller may be constructed by using different structures, it seems difficult to describe a continuous internal model in general. The following definition tries to give an alternative description.

Definition 2: An error-driven controller $u=f(e)$ is said to be internally reducible if, for any matrices $\bar{T}_{21} \in R^{m \times m_{r}}, \bar{T}_{22} \in$ $R^{m \times m_{d}}$ and initial values $x_{r}(0), x_{d}(0)$, there exists an initial controller state $x_{c}(0)$, such that $\bar{T}_{21} x_{r}(t)+\bar{T}_{22} x_{d}(t)=f\left(\Theta_{m}\right)$ for all $t \geq 0$.

By the analysis of transfer function of a linear time-invariant robust servomechanism controller given by Desore and Wang [6], it is obvious that every linear time-invariant error-driven robust servomechanism controller is internally reducible. The advantage of giving the internally-reducible class is that it contains the sampleddata robust servomechanism controller using exponential hold as will be presented in this paper.

\section{INTERNALLY-REDUCIBLE ROBUST SERVOMECHANISMCONTROLLER}

In this section, it is shown that a linear, internally-reducible, errordriven controller is a robust servomechanism controller if the resultant closed-loop system is asymptotically stable. Besides, the steady-state trajectories resulted from a linear, internally-reducible, error-driven robust servomechanism controller is unique. Thus from the viewpoint of "deviation variables" [9], the robust servomechanism problem can be treated as a regulating problem.

\section{A. The Deviation Model}

One of the valuable approaches to synthesize a robust servomechanism controller is by way of the "deviation model." Young and Kwatny [10] have shown that this model can be easily derived from a necessary and sufficient condition for the existence of a robust servomechanism controller stated by Francis [8] and Davison [4]. This model is reviewed and derived briefly as follows.

Let $T_{11} \in R^{n \times m_{r}}, T_{12} \in R^{n \times m_{d}}, T_{21} \in R^{m \times m_{r}}$ and $T_{22} \in$ $R^{m \times m_{d}}$, and define

$$
\begin{aligned}
& x_{s s}(t)=T_{11} x_{r}(t)+T_{12} x_{d}(t) \\
& u_{s s}(t)=T_{21} x_{r}(t)+T_{22} x_{d}(t)
\end{aligned}
$$

and

$$
\begin{gathered}
\delta_{x}(t)=x(t)-x_{s s}(t) \\
\delta_{u}(t)=u(t)-u_{s s}(t) .
\end{gathered}
$$

By substituting (3.b) into the plant (1.a), one obtains

$$
\begin{aligned}
{\left[\begin{array}{c}
\dot{\delta}_{x}(t) \\
e(t)
\end{array}\right]=} & {\left[\begin{array}{ll}
A & B \\
C & O_{m}
\end{array}\right]\left[\begin{array}{l}
\delta_{x}(t) \\
\delta_{u}(t)
\end{array}\right]+\left(\left[\begin{array}{cc}
A & B \\
C & O_{m}
\end{array}\right]\left[\begin{array}{cc}
T_{11} & T_{12} \\
T_{21} & T_{22}
\end{array}\right]\right.} \\
& -\left[\begin{array}{cc}
I_{n} & O_{n \times m} \\
O_{m \times n} & O_{m}
\end{array}\right]\left[\begin{array}{ll}
T_{11} & T_{12} \\
T_{21} & T_{22}
\end{array}\right]\left[\begin{array}{cc}
A_{r} & O_{m_{r} \times m_{d}} \\
O_{m_{d} \times m_{r}} & A_{d}
\end{array}\right] \\
& \left.-\left[\begin{array}{cc}
O_{n \times m_{r}} & -F C_{d} \\
C_{r} & -G C_{d}
\end{array}\right]\right)\left[\begin{array}{l}
x_{r}(t) \\
x_{d}(t)
\end{array}\right]
\end{aligned}
$$

Since eig $\left(A_{r}\right) \subset \mathbb{C}^{+}$, eig $\left(A_{d}\right) \subset \mathbb{C}^{+}$, it is clear from (4) that if $T_{11}, T_{12}, T_{21}$, and $T_{22}$ are chosen to satisfy the matrix equation

$$
\begin{array}{r}
{\left[\begin{array}{cc}
A & B \\
C & O_{m}
\end{array}\right]\left[\begin{array}{ll}
T_{11} & T_{12} \\
T_{21} & T_{22}
\end{array}\right]-\left[\begin{array}{cc}
I_{n} & O_{n \times m} \\
O_{m \times n} & O_{m}
\end{array}\right]\left[\begin{array}{cc}
T_{11} & T_{12} \\
T_{21} & T_{22}
\end{array}\right]} \\
\cdot\left[\begin{array}{cc}
A_{r} & O_{m_{r} \times m_{d}} \\
O_{m_{d} \times m_{r}} & A_{d}
\end{array}\right]=\left[\begin{array}{ccc}
O_{n \times m_{r}} & -F C_{d} \\
C_{r} & -G C_{d}
\end{array}\right]
\end{array}
$$

then the following holds for all $x(0), x_{\mathrm{r}}(0), x_{d}(0)$, and $t \geq 0$ :

$$
\begin{gathered}
\dot{\delta}_{x}(t)=A \delta_{x}(t)+B \delta_{u}(t) \\
e(t)=C \delta_{x}(t)
\end{gathered}
$$

It is proved (see Lemma A.1 in the Appendix) that if and only if the transmission zero assumption (2) is true, then the matrix equation (5) has a unique solution $T_{i j}, i=1,2$ and $j=1,2$. Thus if the transmission zero assumption (2) is true, the model (6) can be uniquely defined.

Theorem 1: A linear, internally-reducible, error-driven controller is a robust servomechanism controller of system (1) if the resultant closed-loop system is asymptotically stable.

Proof: Consider an arbitrary linear, internally-reducible, errordriven controller $u=f(e)$, one claims the following two facts.

Claim 1: Let $T_{11}, T_{12}, T_{21}$, and $T_{22}$ be a solution of (5), and define $\delta_{x}(t)$ and $\delta_{u}(t)$ as (3). If the resultant closed-loop system is asymptotically stable, then it is true that $\delta_{x}(t) \rightarrow 0, \delta_{u}(t) \rightarrow 0$, and $e(t) \rightarrow 0$ as $t \rightarrow \infty$.

Proof: Notice that the internally-reducible condition implies that there exists an initial condition of the controller, such that $\delta_{u}=f(e)$ holds. Hence the asymptotically stable controller can also be considered as a stabilizing regulator of the model (6).

Claim 2: A necessary condition for the closed-loop system to be asymptotically stable is that the transmission zero assumption (2) holds.

Proof: A counter proof will be employed to check this claim. Assume the transmission zero assumption (2) is not true, then by Lemma A.1, there exists a nontrivial solution $T_{i j}, i=1,2$ and $j=1,2$ which satisfy (5) in the case of $F=0, G=0$, and $C_{r}=0$ (i.e., zero disturbance and zero reference input). Now, if the resultant closed-loop system is asymptotically stable, then by Claim 1 , one has $\delta_{x}(t) \rightarrow 0$ and $\delta_{u}(t) \rightarrow 0$ (i.e., $x(t) \rightarrow T_{11} x_{r}(t)+T_{12} x_{d}(t)$ and $\left.u(t) \rightarrow T_{21} x_{r}(t)+T_{22} x_{d}(t)\right)$ as $t \rightarrow \infty$. This is impossible, however, because zero disturbance and zero reference input always implies $x(t) \rightarrow 0$ and $u(t) \rightarrow 0$, as $t \rightarrow \infty$.

Notice that (5) has a unique solution for any parameters $A, B, C, F, G, C_{d}$, and $C_{r}$ if the transmission zero assumption (2) holds. Therefore by the above two claims, it is concluded that the asymptotic tracking action occurs as long as the resultant closed-loop system is asymptotically stable. Hence, the theorem is proved.

Remark 1: Since the transmission zero assumption (2) is a necessary condition for the existence of a robust servomechanism controller and the model (6) can be uniquely defined when the transmission zero assumption holds, so that by the Claim 1 of Theorem 1, it is concluded that $\delta_{x}(t)$ and $\delta_{u}(t)$ are just the deviations from the ultimate steadystate trajectories of $x(t)$ and $u(t)$, respectively. Due to this fact, the model (6) is called the "deviation model" associated with system (1). Notice that the unique existence of deviation model can only be guaranteed in the case of $\operatorname{dim}(u)=\operatorname{dim}(e)$, otherwise, Definition 2 (and so Theorem 1) may be invalid.

\section{B. Steady-State Input Model}

To develop a sampled-data robust servomechanism controller, it is convenient to represent the steady-state input trajectory $u_{s s}(t)$ in terms of a discrete-time model. To do so, let

$$
\lambda(s)=s^{p}-\sum_{i=0}^{p-1} a_{i} s^{i}=0
$$

be the lowest degree polynomial satisfied by $A_{d}$ and $A_{r}$, i.e.,

$$
A_{r}^{p}-\sum_{i=0}^{p-1} a_{i} A_{r}^{i}=0
$$




$$
A_{d}^{p}-\sum_{i=0}^{p-1} a_{i} A_{d}^{i}=0
$$

Define the matrices

$$
\begin{gathered}
\phi=W \Omega W^{-1} \\
\Gamma=\rho W^{-1}
\end{gathered}
$$

where $W \in R^{m p \times m p}$ is a selected nonsingular matrix,

$$
\Omega=\left[\begin{array}{ccccc}
O_{m} & I_{m} & O_{m} & \cdots & O_{m} \\
O_{m} & O_{m} & I_{m} & \cdots & O_{m} \\
\vdots & \vdots & \vdots & \ddots & \\
O_{m} & O_{m} & \cdot & & I_{m} \\
a_{0} I_{m} & a_{1} I_{m} & a_{2} I_{m} & & a_{p-1} I_{m}
\end{array}\right] \in R^{m p \times m p}
$$

and

$$
\rho=\left[I_{m} \mid O_{m \times(p-1)}\right] \in R^{m \times m p} .
$$

From (1.b), (1.c), and (3) it is clear that $u_{s s}(t)$ satisfies the differential equation

$$
\frac{d^{p}}{d t^{p}} u_{s s}(t)-\sum_{i=0}^{p-1} a_{i} \frac{d^{i}}{d t^{i}} u_{s s}(t)=0 .
$$

Thus if one defines

$$
\xi(t)=W\left[\begin{array}{c}
u_{s s}(t) \\
\frac{d}{d t} u_{s s}(t) \\
\vdots \\
\frac{d t^{(p-1)}}{d t^{(p-1)}} u_{s s}(t)
\end{array}\right]
$$

then the steady-state trajectory $u_{s s}(t)$ can be expressed by the following model

$$
\begin{gathered}
\dot{\xi}(t)=\phi \xi(t) \\
u_{s s}(t)=\Gamma \xi(t) .
\end{gathered}
$$

This model can also be transformed to the following discrete-time form

$$
\begin{gathered}
\xi((k+1) T)=\bar{\phi} \xi(k T) \\
u_{s s}(k T+\theta)=\Gamma \exp (\phi \theta) \xi(k T)
\end{gathered}
$$

where $0 \leq \theta<T, T$ is a selected sampling period, and $\bar{\phi}=$ $\exp (\phi T)$.

\section{SAMPLED-Data RobuST SERVOMEChaNISMCONTROLLER}

\section{A. A Linear Dynamical Class}

Consider the following controller (also see Fig. 1):

$$
\begin{aligned}
& {\left[\begin{array}{l}
\hat{\xi}((k+1) T) \\
h((k+1) T)
\end{array}\right]=\left[\begin{array}{cc}
\bar{\phi} & H_{2} \\
O_{g \times m p} & H_{3}
\end{array}\right]\left[\begin{array}{l}
\hat{\xi}(k T) \\
h(k T)
\end{array}\right]+\left[\begin{array}{l}
L_{2} \\
L_{3}
\end{array}\right] e(k T)} \\
& u(k T+\theta)=\left[\Gamma \exp (\phi \theta) \quad \varphi_{h}(\theta)\right]\left[\begin{array}{l}
\xi(k T) \\
h(k T)
\end{array}\right]+\varphi_{e}(\theta) e(k T)
\end{aligned}
$$

where $k=0,1,2, \cdots, H_{2} \in R^{m p \times g}, H_{3} \in R^{g \times g}, L_{2} \in R^{m p \times m}$,

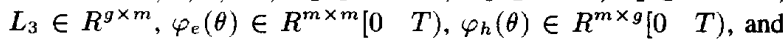
$g$ is a selected nonnegative integer. Now, if the transmission zero assumption (2) holds, one can define

$$
\tilde{\xi}(k T)=\hat{\xi}(k T)-\xi(k T) .
$$

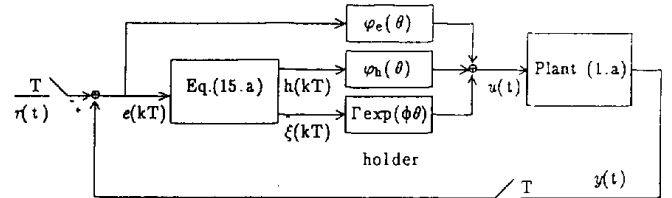

Fig. 1. A dynamical structure of sampled-data robust servomechanism controller using exponential hold.

By subtracting (14) from (15), one obtains

$$
\begin{aligned}
& {\left[\begin{array}{l}
\tilde{\xi}((k+1) T) \\
h((k+1) T)
\end{array}\right]=\left[\begin{array}{cc}
\bar{\phi} & H_{2} \\
O_{g \times m p} & H_{3}
\end{array}\right]\left[\begin{array}{l}
\tilde{\xi}(k T) \\
h(k T)
\end{array}\right]+\left[\begin{array}{l}
L_{2} \\
L_{3}
\end{array}\right] e(k T)} \\
& \delta_{u}(k T+\theta)=\left[\Gamma \exp (\phi \theta) \quad \varphi_{h}(\theta)\right]\left[\begin{array}{l}
\tilde{\xi}(k T) \\
h(k T)
\end{array}\right]+\varphi_{e}(\theta) e(k T) .
\end{aligned}
$$

Thus by combining (17) and the deviation model (6), one obtains the closed-loop sampled-data system

$$
\left[\begin{array}{c}
\delta_{x}((k+1) T) \\
\tilde{\xi}((k+1) T) \\
h((k+1) T)
\end{array}\right]=\left[\begin{array}{ccc}
\bar{A}+L_{1} C & \bar{B}_{\dagger} & H_{1} \\
L_{2} C & \bar{\phi} & H_{2} \\
L_{3} C & O_{g \times m p} & H_{3}
\end{array}\right]\left[\begin{array}{c}
\delta_{x}(k T) \\
\tilde{\xi}(k T) \\
h(k T)
\end{array}\right]
$$

where $\bar{A}, \bar{B}_{\dagger}, L_{1}$ and $H_{1}$ are given by

$$
\begin{gathered}
\bar{A}=\exp (A T) \\
\bar{B}_{\dagger}=\int_{0}^{T} \exp (A \theta) B \Gamma \exp (\phi(T-\theta)) d \theta \\
L_{1}=\int_{0}^{T} \exp (A \theta) B \varphi_{e}((T-\theta)) d \theta \\
H_{1}=\int_{0}^{T} \exp (A \theta) B \varphi_{h}((T-\theta)) d \theta .
\end{gathered}
$$

Theorem 2: If the matrix

$$
\begin{aligned}
{\left[\begin{array}{ccc}
\bar{A} & \bar{B}_{\dagger} & O_{n \times g} \\
O_{m p \times n} & \bar{\phi} & O_{m p \times g} \\
O_{g \times n} & O_{g \times m p} & O_{g \times g}
\end{array}\right] } \\
+\left[\begin{array}{cc}
L_{1} & H_{1} \\
L_{2} & H_{2} \\
L_{3} & H_{3}
\end{array}\right]\left[\begin{array}{ccc}
C & O_{m \times m p} & O_{m \times g} \\
O_{g \times n} & O_{g \times m p} & I_{g}
\end{array}\right]
\end{aligned}
$$

is stable (i.e., all eigenvalues lie insides the unit complex circle), then (15) is a robust servomechanism controller of system (1).

Proof: By the above derivations, one only needs to check that the controller (15) is internally reducible. Notice that for any matrices $\bar{T}_{21} \in R^{m \times m_{r}}, \bar{T}_{22} \in R^{m \times m_{d}}$, and initial values $x_{r}(0), x_{d}(0)$, one can express the function $\bar{T}_{21} x_{r}(t)+\bar{T}_{22} x_{d}(t)$ by the following form (see (11)-(14)):

$$
\begin{aligned}
\eta((k+1) T) & =\bar{\phi} \eta(k T) \\
\bar{T}_{21} x_{r}(t)+\bar{T}_{22} x_{d}(t) & =\Gamma \exp (\phi \theta) \eta(k T)
\end{aligned}
$$

where $\eta(0)=W\left[\eta_{0}^{\tau} \eta_{1}^{\tau} \cdots \eta_{p-1}^{\tau}\right]^{\tau}, \eta_{i}=\bar{T}_{21} A_{r}^{i} x_{r}(0)+$ $\bar{T}_{22} A_{d}^{i} x_{d}(0), i=0,1, \cdots, p-1$, for all $t=k T+\theta, \theta \in\left[\begin{array}{ll}0 & T\end{array}\right)$. Thus by choosing the controller state as $\hat{\xi}(0)=\eta(0), h(0)=0$, and substituting $\{e(k T)\}$ by the zero value sequence, then the controller (15) is reduced to the form (21) (i.e., the controller exists an initial condition such that the zero input $\Theta_{m}$ yields the output $\left.\bar{T}_{21} x_{r}(t)+\bar{T}_{22} x_{d}(t)\right)$. Thus the controller (15) is internally reducible, and the result follows by Theorem 1 . 
Now, by taking $h \equiv 0$, Theorem 1 leads to the following result. Corollary 1 (Minimal-Order Class): If the matrix

$$
\left[\begin{array}{cc}
\bar{A} & \bar{B}_{\dagger} \\
O_{m p \times n} & \bar{\phi}
\end{array}\right]+\left[\begin{array}{l}
L_{1} \\
L_{2}
\end{array}\right]\left[\begin{array}{ll}
C & O_{m \times m p}
\end{array}\right]
$$

is stable, then the following controller

$$
\begin{gathered}
\hat{\xi}((k+1) T)=\bar{\phi} \hat{\xi}(k T)+L_{2} e(k T) \\
u(k T+\theta)=\Gamma \exp (\phi \theta) \hat{\xi}(k T)+\varphi_{e}(\theta) e(k T)
\end{gathered}
$$

is a robust servomechanism controller of system (1).

Remark 2: If $(C, A)$ is detectable and the transmission zero assumption (2) holds, then it is true ([3]-[5]) that

$$
\left(\left[\begin{array}{ll}
C & O_{m \times m p}
\end{array}\right],\left[\begin{array}{cc}
A & B \Gamma \\
O_{m p \times n} & \phi
\end{array}\right]\right) \text { is detectable. }
$$

As a result, for almost the sampling time $T>0$, it is also true that

$$
\left(\left[\begin{array}{ll}
C & O_{m \times m p}
\end{array}\right],\left[\begin{array}{cc}
\bar{A} & \bar{B}_{\dagger} \\
O_{m p \times n} & \bar{\phi}
\end{array}\right]\right) \text { is detectable }
$$

where

$$
\left[\begin{array}{cc}
\bar{A} & \bar{B}_{\dagger} \\
O_{m p \times n} & \bar{\phi}
\end{array}\right]=\exp \left(\left[\begin{array}{cc}
A & B \Gamma \\
O_{m p \times n} & \phi
\end{array}\right] T\right) .
$$

Remark 3: If $(A, B)$ is controllable, then for every specified $L_{1}$ and $H_{1}$, there exist infinitely many choices of $\varphi_{e}(\theta)$ and $\varphi_{h}(\theta)$ to satisfy (19.c) and (19.d), respectively. Thus by the closed-loop sampled-data system (18) (also see (20) or (22)), the parameter choice of the proposed controller (15) (or (23)) can be transformed into the dual of a complete state feedback problem.

\section{B. Continuous-Time Internal Model}

Let us show that the combination of a discrete-time internal model and an exponential hold acts as a continuous-time internal model. Notice that the exponential hold employed in (15.b) is a D/A converter [12] which transforms the digital signals into continuous signals by following the waveform of the exponential function $\Gamma \exp (\phi \theta)$. The pulse response of the exponential hold can be described by

$$
E P(\theta)=\left[\begin{array}{ll}
\Gamma \exp (\phi \theta) & : 0 \leq \theta<T \\
O_{m \times m p} & : \text { otherwise }
\end{array} .\right.
$$

Thus the exponential hold has a transfer function matrix

$$
\begin{aligned}
H_{e p}(s) & =\int_{0}^{T} e^{-\theta s} \Gamma \exp (\phi \theta) d \theta \\
& =\Gamma\left(I_{m p}-e^{-T s} \exp (\phi T)\right)\left(s I_{m p}-\phi\right)^{-1} \\
& =\Gamma\left(I_{m p}-z^{-1} \bar{\phi}\right)\left(s I_{m p}-\phi\right)^{-1}
\end{aligned}
$$

where the term $\left(I_{m p}-z^{-1} \bar{\phi}\right)$ can be canceled by the transfer function of the discrete-time internal model contained in (15.a), and the left term $\left(s I_{m p}-\phi\right)$ is just a continuous-time internal model.

\section{EXAMPLE}

Consider a de motor described by equation [5].

$$
\begin{gathered}
\dot{x}(t)=\left[\begin{array}{cc}
-\frac{B_{c}}{J} & \frac{K_{t}}{J} \\
-\frac{K_{c}}{L} & -\frac{R}{L}
\end{array}\right] x(t)+\left[\begin{array}{l}
0 \\
\frac{1}{L}
\end{array}\right] u(t)+\left[\begin{array}{r}
-\frac{1}{J} \\
0
\end{array}\right] d \\
y(t)=\left[\begin{array}{ll}
1 & 0
\end{array}\right] x(t)
\end{gathered}
$$

where $d=1$ is a constant disturbance. The output is desired to track a sinusoidal signal described as

$$
\dot{x}_{r}(t)=\left[\begin{array}{rr}
0 & -\omega \\
\omega & 0
\end{array}\right] x_{r}(t), \quad x_{r}(0)=\left[\begin{array}{r}
0 \\
-1
\end{array}\right]
$$

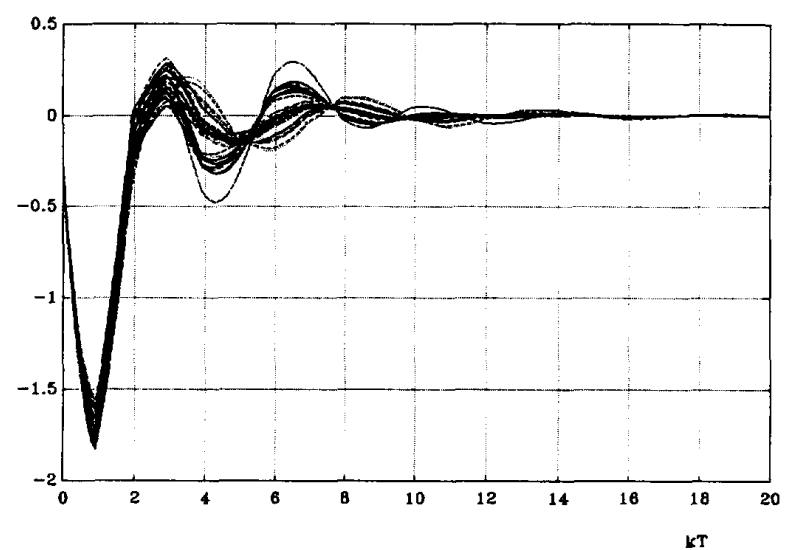

Fig. 2. Robust illustration of sampled-data controller (27), where 40 tracking error responses are plotted with parameters randomly varied over a range of $\pm 25 \%$ for $R, L, B_{e}$, and $J$.

$$
r(t)=\left[\begin{array}{ll}
1 & 0
\end{array}\right] x_{r}(t)
$$

Let $T=0.5 \pi / \omega$ and

$$
\phi=\left[\begin{array}{rrr}
0 & 0 & 0 \\
0 & 0 & -\omega \\
0 & \omega & 0
\end{array}\right], \quad \Gamma=\left[\begin{array}{lll}
1 & 1 & 0
\end{array}\right] .
$$

Then a sampled-data robust servomechanism controller of (23) is given as

$$
\hat{\xi}((k+1) T)=\left[\begin{array}{rrr}
1 & 0 & 0 \\
0 & 0 & -1 \\
0 & 1 & 0
\end{array}\right] \hat{\xi}(k T)+L_{2} e(k T)
$$

$$
u(k T+\theta)=L_{0} e(k T)+\left[\begin{array}{ll}
1 & \cos (\omega \theta)-\sin (\omega \theta)
\end{array}\right] \hat{\xi}(k T) .
$$

Assume the parameters of the dc motor are $B_{e}=0.0162, J=$ $0.215, K_{t}=K_{e}=1.11, R=1.05, L=0.0053$, and $\omega=5$. If one chooses $L_{0}=-0.5, L_{2}=[-0.5,0.5,-0.5]^{\top}$, then from (22), one has

$$
\begin{aligned}
\operatorname{eig}\left(\left[\begin{array}{cc}
\bar{A}+L_{1} C & \bar{B}_{\dagger} \\
L_{2} C & \bar{\phi}
\end{array}\right]\right)=\{0.0327, & 0.1591 \pm 0.5541 i \\
& \pm 0.2242 \pm 0.3907 i\} .
\end{aligned}
$$

Thus (27) is a robust servomechanism controller of the dc motor. The robust property of this controller has been illustrated by the simulation shown in Fig. 2 (where 40 curves are plotted with parameters randomly varied over a range of $\pm 25 \%$ for $R, L, B_{e}$, and $J$ ). To show the advantage of using an exponential hold, the following digital controller using zero-order hold (the closed-loop poles are $0,0.4254 \pm 0.6642 i, 0.1581 \pm 0.5009 i)$ is also simulated for comparison (notice that the comparison is only for the convenience of illustration of ripple error)

$$
\begin{gathered}
\hat{\xi}((k+1) T)=\left[\begin{array}{rrr}
1 & 0 & 0 \\
0 & 0 & -1 \\
0 & 1 & 0
\end{array}\right] \hat{\xi}(k T)+\left[\begin{array}{r}
-0.5 \\
0.5 \\
0.5
\end{array}\right] e(k T) \\
u(k T+\theta)=\left[\begin{array}{lll}
1 & 1 & 0
\end{array}\right] \hat{\xi}(k T) .
\end{gathered}
$$

The sinusoidal tracking responses by using the controllers (27) and (29) are shown in Fig. 3 and Fig. 4 

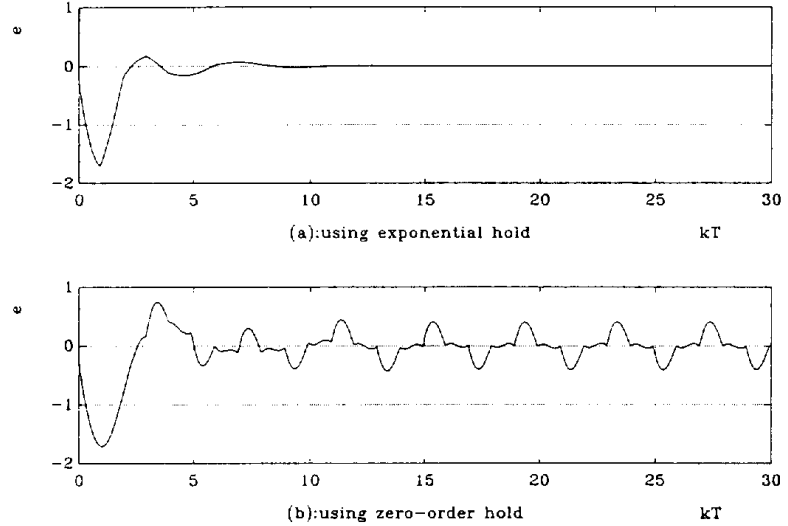

Fig. 3. Comparison of tracking error responses, where (a) using the sampled-data controller (27), (b) using the sampled-data controller (29).
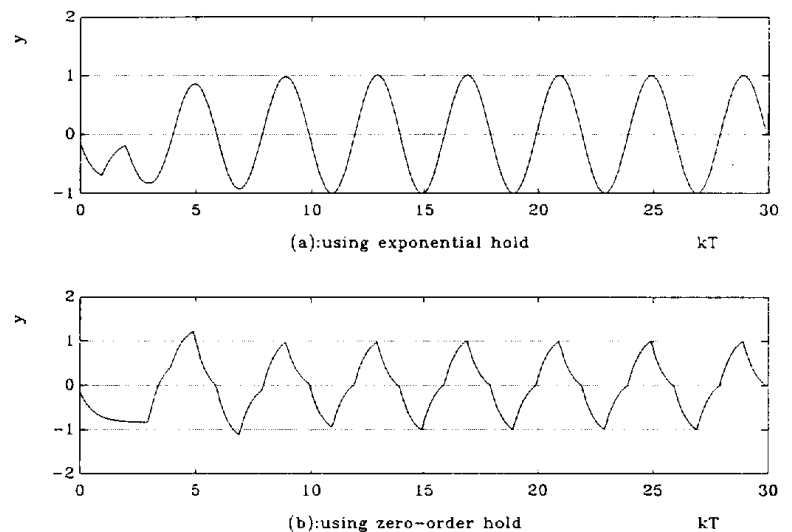

Fig. 4. Comparison of output responses, where (a) using the sampled-data controller (27), (b) using the sampled-data controller (29).

\section{CONCLUSIONS}

In this paper, a new strategy based on the combination of a discrete-time internal model and an exponential hold is proposed for constructing a ripple free sampled-data robust servomechanism controller. The proposed controller can guarantee the asymptotic tracking not only at, but also between, the sampling instances. This is due to the weighting by the exponential hold, so that the correct waveform of input can be obtained when the steady state is reached.

An advantage of this approach is that more design freedoms can be obtained from the structure. Thus even if the controller is at the simplest form, it remains possible to convert the design into the dual of a complete state feedback problem (see Corollary 1). Besides, the measurements and the on-line computations of the proposed controller are arranged to base on the sampling periods, so that the control scheme is easily implemented by digital computers.

\section{APPENDIX}

Lemma A.1: Let $E_{1} \in C^{q \times q}, E_{2} \in C^{q \times q}, E_{3} \in C^{f \times f}$ and $E_{4} \in C^{q \times f}$, then there exists a unique solution $M \in C^{q \times f}$ for the matrix equation

$$
E_{1} M+E_{2} M E_{3}=E_{4}
$$

if and only if $E_{1}+s E_{2}$ is nonsingular for all $s \in \operatorname{eig}\left(E_{3}\right)$.
Proof: One can rearrange (A.1) as the equivalent form

$$
E_{1} \bar{M}+E_{2} \overline{M E}_{3}=\bar{E}_{4}
$$

where $\bar{M}=M V, \bar{E}_{3}=V^{-1} E_{3} V, \bar{E}_{4}=E_{4} V$, and $V \in C^{f \times f}$ is a nonsingular matrix which is chosen such that $\bar{E}_{3}$ is lower triangular. Now, assume $\bar{M}=\left[M_{1} M_{2} \cdots M_{f}\right], \bar{E}_{4}=\left[E_{41} E_{42} \cdots E_{4 f}\right]$, and

$$
\bar{E}_{3}=\left[\begin{array}{cccc}
s_{11} & 0 & \cdots & 0 \\
s_{21} & s_{22} & \cdots & 0 \\
\vdots & \vdots & & \vdots \\
s_{f 1} & s_{f 2} & \cdots & s_{f f}
\end{array}\right]
$$

where $M_{i} \in C^{q \times 1}$ and $E_{4 i} \in C^{q \times 1}(i=1,2, \cdots, f)$ are the columns of $\bar{M}$ and $\bar{E}_{4}$, respectively. By comparing the multiplication of each column, (A.2) can be further rearranged as the following Kronecker product [13] form

$$
\begin{array}{r}
\left(\left[\begin{array}{cccc}
E_{1} & O_{q} & \cdots & O_{q} \\
O_{q} & E_{1} & \cdots & O_{q} \\
\vdots & \vdots & \ddots & \vdots \\
O_{q} & O_{q} & \cdots & E_{1}
\end{array}\right]+\left[\begin{array}{cccc}
s_{11} E_{2} & s_{21} E_{2} & \cdots & s_{f 1} E_{2} \\
O_{q} & s_{22} E_{2} & \cdots & s_{f 2} E_{2} \\
\vdots & \vdots & \ddots & \vdots \\
O_{q} & O_{q} & \cdots & s_{f f} E_{2}
\end{array}\right]\right) \\
\\
\cdot\left[\begin{array}{c}
M_{1} \\
M_{2} \\
\vdots \\
M_{f}
\end{array}\right]=\left[\begin{array}{c}
E_{41} \\
E_{42} \\
\vdots \\
E_{4 f}
\end{array}\right] .
\end{array}
$$

Thus by checking the diagonal terms, the lemma is easily derived.

\section{REFERENCES}

[1] G. F. Franklin and A. Emami-Naeini, "Design of ripple-free multivariable robust servomechanisms," IEEE Trans. Automat. Contr., vol. AC-31, pp. 661-664, 1986.

[2] R. Doraiswami, "Robust control strategy for a linear time-invariant multivariable sampled-data servomechanism problem," in IEE Proc., vol. 129,1982 , pp. 283-292.

[3] E. J. Davison and A. Goldenberg, "Robust control of a general servomechanism problem: The servo compensator," Automatica, vol. 11, pp. $461-471,1975$.

[4] E. J. Davison, "The robust control of a servomechanism problem for linear time-invariant multivariable system," IEEE Trans. Automat. Contr., vol. AC-21, pp. 25-34, 1976.

[5] E. J. Davison and I. J. Ferguson, "The design of controllers for the multivariable robust servomechanism problem using parameter optimization method," IEEE Trans, Automat. Contr., vol. AC-26, pp. 93-110, 1981.

[6] C. A. Desore and Y. T. Wang, "On the minimal order of a robust servocompensator," IEEE Trans. Automat. Contr., vol. AC-23, pp. 70-73, 1978.

[7] C. A. Desore and Y. T. Wang, "Linear time-invariant robust servomechanism problem: A self-contained exposition," in Control and Dynamical System, vol. 16, C. T. Leondes, Ed. New York: Academic, 1980.

[8] B. A. Francis and W. M. Wonham, "The internal model principle of control theory," Automatica, vol. 12, pp. 457-465, 1976.

[9] K. D. Young, "Deviation variables in linear multivariable servomechanisms," IEEE Trans. Automat. Contr., vol. AC-29, pp. 567-569, 1984.

[10] K. D. Young and H. G. Kwatny, "Variable structure servomechanism design and applications to overspeed protection control," Automatica, vol. 18 , pp. $385-400,1982$.

[11] N. H. Rosenbrock, Computer-Aided Control System Design. New York: Academic, 1974.

[12] B. C. Kuo, Digital Control System. New York: Holt, Rinehart and Winston, 1980.

[13] T. Kailath, Linear System. Englewood Cliffs, NJ: Prentice-Hall, 1980. 